\title{
Sinais e Sistemas Definidos sobre Aritmética Intervalar Complexa
}

F.T. SANTANA ${ }^{1}$, Instituto Federal de Educação, Ciência e Tecnologia do Rio Grande do Norte, Currais Novos, RN, Brasil.

F.L. SANTANA, A.D. DÓRIA NETO, R.H.N. SANTIAGO ${ }^{2}$, UFRN, Campus Universitário Lagoa Nova, 59078-970 Natal, RN, Brasil.

Resumo. Neste trabalho, é feita a fundamentação para os conceitos de sinais e sistemas intervalares complexos, fazendo-se o uso da aritmética complexa retangular e do conceito de intervalo de números complexos feito com auxílio da chamada ordem de Kulisch-Miranker para complexos. É apresentado também o conceito de representação intervalar e é definida a representação canônica intervalar (CIR) de funções complexas. A partir de um sistema $f$ que opere sobre sinais cujos valores sejam números complexos, usando a função CIR, encontra-se um sistema intervalar $F$ o qual preserva, no ambiente intervalar, as propriedades de $f$, tais como estabilidade, invariância no tempo, aditividadade, homogeneidade e linearidade.

Palavras-chave. Aritmética Complexa Intervalar, Intervalos de Complexos, Sinais Intervalares, Sistemas Intervalares, Representação Intervalar.

\section{Introdução}

Um dos tópicos de interesse da área de processamento digital de sinais (PDS) é o problema de representação de sistemas reais em hardware e/ou software [3, 10, 21]. Tal representação é passível de erros e imprecisões, provocados por falhas nos instrumentos de medição, limitações de representação em máquina, etc. Como forma de controlar tais erros e imprecisões os autores sugerem o uso da matemática intervalar.

A matemática intervalar lida com dados numéricos na forma de intervalos compactos com o objetivo de codificar os erros computacionais, ou imprecisões, no próprio intervalo $[7,11,12,13]$. Por isso, a abordagem intervalar de algumas ferramentas usadas em PDS, como sinais, sistemas e transformadas proporciona maior controle de erros nas representações e também resultados confiáveis nas implementações de cálculos computacionais complexos, como as convoluções [15].

Os sinais utilizados em aplicações, na maior parte dos casos, são contínuos no tempo. Por exemplo, na Figura 1, tem-se um circuito LC paralelo com indutor $L$ e capacitor $C$ supostos ideais. A operação desse circuito é descrita pela equação

\footnotetext{
${ }^{1}$ fabiana.santana@ifrn.edu.br

${ }^{2}$ fagner@ccet.ufrn.br, adriao@dca.ufrn.br, regivan@dimap.ufrn.br
} 
diferencial de segunda ordem $L C \frac{d^{2} v(t)}{d t^{2}}+v(t)=0$, em que $v(t)$ é a tensão no capacitor no tempo $t, C$ é sua capacitância e $L$ é a indutância do indutor. Para $v(0)=V_{0}$ a solução dessa equação é o sinal $v(t)=V_{0} \cos \left(\omega_{0} t\right), t \geq 0$, em que $\omega_{0}=\frac{1}{\sqrt{L C}}[6]$. Supondo $V_{0}=\sqrt{3}$ e $\omega_{0}=5 \pi$, segue que $v(t)=\sqrt{3} \cos (5 \pi t)$.

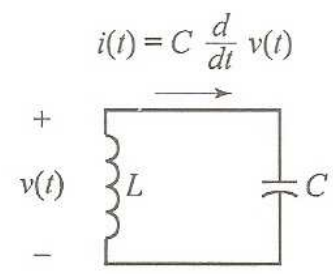

Figura 1: Haykin [6]. Circuito LC paralelo com indutor $L$ e capacitor $C$ ideais.

Para processar o sinal $v(t)=\sqrt{3} \cos (5 \pi t)$ computacionalmente é necessário fazer a amostragem do mesmo. Por exemplo, considerando $t \in \mathbb{N}, 1 \leq t \leq N$, a amostragem do sinal $v(t)$ é composta pelos valores $v(1), \cdots, v(N)$. Como o sistema computacional não representa todos os números reais, é comum que alguns dos valores $v(t), 1 \leq t \leq N$ seja um valor com erro ou imprecisão. A matemática intervalar possibilita representar os valores com imprecisão por intervalos. Para ilustrar, suponha que o sistema computacional tenha precisão de dois dígitos decimais. Nesse sistema o valor $v(1)=\sqrt{3} \cos (5 \pi)=1.667365859 \ldots$, que antes era representado por 1.67 , será representado pelo valor intervalar [1.66,1.67], pois 1.66 é o maior valor menor ou igual a $v(1)$ com dois dígitos decimais e 1.67 é o menor valor maior ou igual a $v(1)$ com dois dígitos decimais.

Em vários trabalhos, como $[2,3,4,5,10,18]$, encontram-se estudos do processamento digital de sinais com o uso da matemática intervalar. Os autores estão adotando os intervalos para capturar a imprecisão existente no processo de representação numérica ou gerada durante o processamento. Essa alternativa tem se mostrado uma solução para a perda das informações, pois a representação intervalar armazena a informação do quão imprecisa é tal representação.

Em todos esses trabalhos, intuitivamente, utiliza-se sinais intervalares, o que motiva a fundamentação das noções de sinais e sistemas intervalares, assim como de suas propriedades, da mesma forma que é feito para o caso clássico dos sinais complexos.

Este trabalho é organizado como segue. Na Seção 2, é abordada uma aritmética complexa intervalar. Na Seção 3, são definidos sinais intervalares complexos e mostrado que um sinal intervalar complexo se escreve como combinação de impulsos deslocados, como acontece no caso clássico. Na Seção 4, são definidas as principais classes de sistemas complexos intervalares, tais como: invariância no tempo, estabilidade, aditividade, homogeneidade e linearidade. Além disso, faz-se a extensão do conceito de representação canônica intervalar (CIR), definida em [7], para o caso complexo, que associa imagens de funções complexas sujeitas a imprecisões à números complexos intervalares, capturando a imprecisão no sinal. Utiliza-se a função 
CIR para estender sistemas clássicos a sistemas complexos intervalares e mostra-se que dessa forma as propriedades citadas acima são preservadas.

\section{Aritmética Retangular}

Em 1968, Alefeld [1] introduziu a representação retangular para números complexos sujeitos a imprecisões e uma aritmética que, sob certas condições, reduz-se à aritmética de Moore para intervalos reais [12]. Algumas de suas propriedades, como a corretude e a inclusão monotônica, estão demonstradas em [1], onde os autores também observam que as operações de multiplicação e divisão introduzem certo grau de imprecisão, mas, apesar disso, apresentam resultados satisfatórios com respeito a aplicações [10, 17]. Pode-se encontrar mais propriedades a respeito da aritmética complexa intervalar em [16].

Definição 2.1. (Conjunto dos Intervalos de Números Reais) Sejam $\underline{a}, \bar{a} \in \mathbb{R}$, com $\underline{a} \leq \bar{a}$. O intervalo $[\underline{a}, \bar{a}]$ é constituído por todo $a \in \mathbb{R}$ tal que $\underline{a} \leq a \leq \bar{a}$.

Observação 1. O conjunto dos intervalos reais é denotado por $\mathbb{I}$.

Definição 2.2. (Aritmética de Moore em $\mathbb{I R}$ ) Dados os intervalos reais $A=[\underline{a}, \bar{a}]$ e $B=[\underline{b}, \bar{b}]$ as operações elementares são definidas por:

(a) $A+B=[\underline{a}+\underline{b}, \bar{a}+\bar{b}]$;

(b) $A-B=[\underline{a}-\bar{b}, \bar{a}-\underline{b}]$;

(c) $A \times B=[\min \{\underline{a b}, \underline{a} \bar{b}, \bar{a} \underline{b}, \bar{a} \bar{b}\}, \max \{\underline{a b}, \underline{a} \bar{b}, \bar{a} \underline{b}, \bar{a} \bar{b}\}]$;

(d) $\frac{A}{B}=A \times B^{-1}=\left[\min \left\{\frac{a}{\underline{b}}, \frac{a}{\bar{b}}, \frac{\bar{a}}{\underline{b}}, \frac{\bar{a}}{\bar{b}}\right\}, \max \left\{\frac{\underline{a}}{\underline{b}}, \frac{a}{\bar{b}}, \frac{\bar{a}}{\underline{b}}, \frac{\bar{a}}{\bar{b}}\right\}\right]$, desde que $0 \notin B$.

O conjunto destas operações é o que se chama aritmética de Moore sobre $\mathbb{I R}$.

Definição 2.3 (Distância para Intervalos Reais). Sejam $A=[\underline{a}, \bar{a}], B=[\underline{b}, \bar{b}] \in \mathbb{I} \mathbb{R}$. $A$ distância intervalar entre $A$ e $B$ é definida por $d_{M}(A, B)=\max \{|\underline{a}-\underline{b}|,|\bar{a}-\bar{b}|\}$, em que $|a|$ é o módulo de a.

Definição 2.4 (Valor Absoluto de Intervalos Reais). Seja $A=[\underline{a}, \bar{a}] \in \mathbb{R}$. Definese o valor absoluto de $A$ por $|A|_{R}=d_{M}(A,[0,0])=\max \{|\underline{a}|,|\bar{a}|\}$.

Definição 2.5 (Número Complexo Intervalar). Sejam $A=[\underline{a}, \bar{a}], B=[\underline{b}, \bar{b}] \in \mathbb{R}$. Um número complexo intervalar retangular [1] ou simplesmente número complexo intervalar $Z$ é definido como $Z=A+i B=\{z=a+i b: a \in A, b \in B\}$.

Observação 2. O número complexo intervalar $Z=A+i B$ constitui um retângulo no plano complexo com lados paralelos aos eixos coordenados. $O$ conjunto dos números complexos intervalares será denotado por $R(\mathbb{C})$.

Definição 2.6 (Aritmética Retangular). Sejam $A, B, C, D \in \mathbb{R} e\{+,-, \times, /\} o$ conjunto das operações aritméticas em $\mathbb{R}$. Para $X=A+i B, Y=C+i D \in R(\mathbb{C})$ a aritmética intervalar complexa, ou aritmética retangular, é definida por: 
(a) $X \oplus_{R} Y=(A+C)+i(B+D)$;

(b) $X \ominus_{R} Y=(A-C)+i(B-D)$;

(c) $X \otimes_{R} Y=(A C-B D)+i(A D+B C)$;

(d) $X \oslash_{R} Y=(A C+B D) /\left(C^{2}+D^{2}\right)+i(B C-A D) /\left(C^{2}+D^{2}\right)$, desde que $0 \notin\left(C^{2}+D^{2}\right)$.

Note que para $a \in \mathbb{R}, a Y=([a, a]+i[0,0]) \otimes_{R} Y=[a, a] C+i[a, a] D$. Por simplicidade utilizaremos $a Y=a C+i a D$.

Proposição 2.1. Para $X, Y, Z \in R(\mathbb{C})$ são válidas as seguintes propriedades:

(a) $X \oplus_{R} Y=Y \oplus_{R} X$ e $X \otimes_{R} Y=Y \otimes_{R} X$;

(b) $\left(X \oplus_{R} Y\right) \oplus_{R} Z=X \oplus_{R}\left(Y \oplus_{R} Z\right)$;

(c) $X=[0,0]+i[0,0]$ e $Y=[1,1]+i[0,0]$ são os elementos neutros da adição $\oplus_{R}$ e multiplicação $\otimes_{R}$, respectivamente;

(d) $X \otimes_{R}\left(Y \oplus_{R} Z\right) \subseteq\left(X \otimes_{R} Y\right) \oplus_{R}\left(X \otimes_{R} Z\right)$;

(e) $x \otimes_{R}\left(Y \oplus_{R} Z\right)=\left(x \otimes_{R} Y\right) \oplus_{R}\left(x \otimes_{R} Z\right)$, para $x \in \mathbb{C}$.

Demonstração. A demonstração pode ser vista em [1].

Definição 2.7 (Distância para Números Complexos Intervalares). Sejam $A, B, C, D \in$ $\mathbb{I} \mathbb{R}$ e $X=A+i B, Y=C+i D \in R(\mathbb{C})$. A distância intervalar entre $X$ e $Y$ é definida por $D_{M}(X, Y)=d_{M}(A, C)+d_{M}(B, D)$.

Definição 2.8 (Valor Absoluto de Números Complexos Intervalares). Sejam A, B $\mathbb{I}$ e $X=A+i B \in R(\mathbb{C})$. Define-se o valor absoluto de $X$ por $|X|_{C}=d_{M}(A,[0,0])+$ $d_{M}(B,[0,0])$.

\section{Sinais Intervalares Complexos}

A noção de ambiguidade possui diferentes significados, de acordo com Mukaidomo ([14] p. 12) eles são classificados em: incompleto, ambiguidade, aleatoriedade, imprecisão e nebulosidade. Este trabalho lida com o caso da imprecisão em PDS que geralmente envolve erros ${ }^{3}$ ou ruídos [15]. Na abordagem aqui proposta, o valor do sinal, num instante $n$, é representado por um intervalo. A vantagem disto está no fato de que o próprio intervalo informa, através da sua amplitude, o erro nele contido, portanto ao término de um processamento intervalar é possível se detectar a quantidade de imprecisão existente no processamento pela simples análise dos valores de saída.

Definição 3.1 (Sinal Intervalar). Um sinal intervalar a tempo discreto é definido como uma sequência $X: \mathbb{Z} \rightarrow \mathbb{R}$. Usa-se a notação $X[n]$ para representar o valor do sinal em $n \in \mathbb{Z}$, ou o próprio sinal $X$.

\footnotetext{
${ }^{3} \mathrm{Um}$ sinal digital é um sinal discreto, cuja amplitude assume valores num conjunto discreto. Normalmente esse tipo de sinal se origina da quantização de um sinal discreto com valores num conjunto contínuo. Isto é, para cada amostra, a amplitude do sinal é aproximada para o valor numérico correspondente ao nível de quantização mais próximo e, com isso, origina-se também um erro de quantização.
} 
Definição 3.2 (Sinal Intervalar Complexo). Sejam $A[n], B[n] \in \mathbb{R}$ dois sinais intervalares. Um sinal intervalar complexo a tempo discreto é uma seqüência $X$ : $\mathbb{Z} \rightarrow R(\mathbb{C})$ que associa para cada $n \in \mathbb{Z}$ um número complexo intervalar $X[n]=$ $A[n]+i B[n] \in R(\mathbb{C})$.

Para obter amostras intervalares de um sinal contínuo serão utilizadas as funções maior inteiro, $\lfloor x\rfloor: \mathbb{R} \rightarrow \mathbb{Z}$, definida por $\lfloor x\rfloor=\sup \{z \in \mathbb{Z} \mid z \leq x\}$ e menor inteiro, $\lceil x\rceil: \mathbb{R} \rightarrow \mathbb{Z}$, definida por $\lceil x\rceil=\inf \{z \in \mathbb{Z} \mid z \geq x\}$.

Exemplo 1. Para processar computacionalmente o sinal discreto no tempo $x(n)=$ $(0.9)^{n}\left\{0.5 \cos (0.2 \pi n)-\frac{\sqrt{3}}{2} \sin (0.2 \pi n)\right\}$, primeiramente, é necessário obter as amostras do sinal. Para obter as amostras intervalares do sinal dado, serão feitas as seguintes considerações. Seja $\mathbb{F}$ o conjunto de todos os intervalos cujos extremos pertencem a um sistema de ponto flutuante $F, \epsilon$ o menor número representável em $F, R: \mathbb{R} \rightarrow \mathbb{F}$ a função definida por $R(x)=[\underline{x}, \bar{x}]$, em que $\underline{x}=\lfloor x / \epsilon\rfloor * \epsilon \in F$ é o maior número menor ou igual a $x$ e $\bar{x}=\lceil x / \epsilon\rceil * \epsilon \in F$ é o menor número maior ou igual a $x$ e $X[n]=R(x(n))$ representa as amostras intervalares [19]. Considerando $F$ um sistema de ponto flutuante com dois dígitos decimais e $\epsilon=0.01$, para $0 \leq n \leq 10$, obtém-se as seguintes amostras intervalares: $X[0]=[0.50,0.50]$, $X[1]=[-0.10,-0.09], X[2]=[-0.55,-0.54], X[3]=[-0.72,-0.71], X[4]=$ $[-0.60,-0.59], X[5]=[-0.30,-0.29], X[6]=[0.05,0.06], X[7]=[0.32,0.33]$, $X[8]=[0.42,0.43], X[9]=[0.35,0.36], X[10]=[0.17,0.18]$.

Um sinal discreto clássico pode ser representado por combinação linear de impulsos deslocados, onde a função impulso unitário definida por:

$$
\delta(n)= \begin{cases}1, & \text { se } n=0 \\ 0, & \text { caso contrário. }\end{cases}
$$

é utilizada para tal. Esse fato foi estendido em [21, 22] para o caso de sinais cujos valores são intervalos de extremos reais e é estendido abaixo para o caso em que os valores são intervalos de extremos complexos. Essa propriedade possibilita a compreensão e análise do comportamento do sinal e, a partir dela, pode-se definir a transformada $\mathcal{Z}$ de um sinal discreto. A transformada $\mathcal{Z}$ é uma ferramenta importante na análise de sistemas discretos no tempo. Um dos fatores que a caracteriza é o fato de ao se aplicar uma função exponencial na entrada de sistemas lineares e invariantes no tempo obtém-se como resposta uma fução exponencial do mesmo tipo, porém com amplitude modificada [15].

Lema 3.1. Todo sinal intervalar complexo $X[n]$, discreto no tempo, é representado por combinação linear de impulsos deslocados, isto é $X[n]=\sum_{k=-\infty}^{\infty} X[k] \delta(n-k)$

Demonstração. A demonstração é imediata, basta considerar que $\delta(n-k) \neq 0$ apenas em $k=n$.

Observação 3. Aplicando o resultado da Lema 3.1, para $0 \leq n \leq 10$, a representação intervalar do sinal $x(n)=(0.9)^{n}\left\{0.5 \cos (0.2 \pi n)-\frac{\sqrt{3}}{2} \sin (0.2 \pi n)\right\}$, dado no 
Exemplo 1, é: $X[n]=X[0] \delta(n)+X[1] \delta(n-1)+X[2] \delta(n-2)+X[3] \delta(n-3)+$ $X[4] \delta(n-4)+X[5] \delta(n-5)+X[6] \delta(n-6)+X[7] \delta(n-7)+X[8] \delta(n-8)+X[9] \delta(n-$ $9)+X[10] \delta(n-10)$.

\section{Sistemas Intervalares Complexos}

Em PDS os sinais são processados por sistemas, que são operadores que podem modificá-los ou extrair informação [9]. Em outras palavras, um sistema é uma entidade que processa um conjunto de sinais (entradas) resultando em um outro conjunto de sinais (saídas). Tais sistemas podem ser fisicamente realizáveis ou não. Em [21], encontram-se as primeiras idéias de sistemas intervalares reais, além de algumas propriedades. Porém, ainda há uma carência de fundamentação teórica. Aqui, será estabelecida a fundamentação matemática para o caso complexo e feita a extensão de sistemas clássicos a sistemas intervalares, para isso este trabalho estende a noção de representação canônica intervalar, proposta em [20], para o caso complexo.

Definição 4.1 (Sistema Intervalar Complexo). Um sistema intervalar complexo em tempo discreto é uma função $F: R(\mathbb{C}) \rightarrow R(\mathbb{C})$, ou seja, é um operador que mapeia sequências de intervalos complexos $X[n]$ em outra sequência de intervalos complexos $Y[n]$. Isto é, dado um sinal $X[n]$ (sinal de entrada) o sistema retorna um o sinal $Y[n]=F(X[n])$ (sinal de saída).

Dependendo das propriedades do operador $F$, o sistema intervalar complexo pode ser classificado em estável, invariante no tempo e linear, dentre outras propriedades, como ausência de memória, passividade, ausência de perdas [15]. No que se segue, define-se estes conceitos no caso de sistemas intervalares complexos.

Definição 4.2 (Sistemas Causais). Um sistema intervalar complexo $F$ é dito ser causal se, quando $X[n]=Y[n]$ para $n<n_{0}$, então $F(X[n])=F(Y[n])$, para $n<n_{0}$.

Definição 4.3 (Sistemas Estáveis). Um sistema intervalar complexo $F$ é estável se, para todo sinal de entrada $X[n]$ limitado, isto é, para todo $n \in \mathbb{Z},|X[n]|_{C}<K_{1}$, para algum $0<K_{1}<\infty$, o sinal de saída $F(X[n])$ também é limitado, isto é, para todo $n \in \mathbb{Z},|F(X[n])|_{C}<K_{2}$, para algum $0<K_{2}<\infty$.

Definição 4.4 (Sistemas Invariantes no Tempo). Um sistema intervalar complexo é invariante no tempo se, para qualquer sinal de entrada $X[n]$ e qualquer inteiro $n_{0}, F\left(X\left[n-n_{0}\right]\right)=Y\left[n-n_{0}\right], \operatorname{com} Y[n]=F(X[n])$.

Definição 4.5 (Sistemas Lineares). Um sistema intervalar complexo é linear se e somente se $F(a X[n])=a X[n]$ e $F(X[n]+Y[n])=F(X[n])+F(Y[n])$, para qualquer número real a e quaisquer sinais intervalares complexos $X[n]$ e $Y[n]$.

Na próxima seção, apresenta-se o conceito de representação canônica intervalar para o caso complexo. A partir desse conceito, demonstra-se que sistemas pontuais estendidos via representação canônica preservam as propriedades definidas acima. 


\subsection{Representação e extensão de sistemas complexos}

A motivação inicial da matemática intervalar é a de trabalhar com dados intervalares que representam dados numéricos com imprecisões e/ou erros. É muito importante que os dados intervalares usados sejam corretos, no sentido de que cada intervalo contenha o número real que ele representa. Baseado nisto, tem-se o conceito de representação intervalar.

Definição 4.6 (Representação Intervalar, [20]). Dado um número real $a \in \mathbb{R}$ e um intervalo $[x, y]$, diz-se que $[x, y]$ representa a quando $a \in[x, y]$.

Definição 4.7 (Representação Intervalar de Funções Reais, [20]). Uma função intervalar $F: \mathbb{I} \rightarrow \mathbb{R}$ representa a função real $f: \mathbb{R} \rightarrow \mathbb{R}$ quando para todo $x \in[a, b]$ tem-se $f(x) \in F([a, b])$.

Em [20], os autores definem a representação canônica intervalar (CIR) de funções reais, da seguinte forma: se $f: \mathbb{R} \rightarrow \mathbb{R}$ é uma função que não possui assíntotas verticais (ou seja, não existe $a \in \mathbb{R}$ tal que $\lim _{x \rightarrow a^{ \pm}} f(x)= \pm \infty$ ), então sua representação canônica intervalar é definida como sendo a função $C I R(f): \mathbb{R} \rightarrow \mathbb{R}$ tal que $C I R(f)([a, b])=[\min f([a, b]), \max f([a, b])]$. No mesmo artigo, é mostrado que se $f$ é contínua em um intervalo $[a, b]$, então $C I R(f)([a, b])=f([a, b])$, em que $f([a, b])$ representa a imagem do conjunto $[a, b]$ pela função $f$ e que a função $C I R(f)$ é a melhor representação intervalar para funções reais, no sentido de que qualquer outra representação intervalar $F$ para a função $f$ satisfaz $C I R(f)([a, b]) \subseteq F([a, b])$. Aqui, esses conceitos serão estendidos para o caso complexo, a fim de encontrar a melhor representação intervalar para funções complexas.

Definição 4.8 (Representação Intervalar de Números Complexos). Dado um número complexo $z=a+i b \in \mathbb{C}$ e um número complexo intervalar $Z=A+i B \in R(\mathbb{C})$, diz-se que $Z$ representa $z$ se $a \in A$ e $b \in B$.

Definição 4.9 (Representação Intervalar de Funções Complexas). Sejam $z=a+$ $i b \in \mathbb{C}, Z=A+i B \in R(\mathbb{C}), f: \mathbb{C} \rightarrow \mathbb{C}$ uma função complexa e $F: R(\mathbb{C}) \rightarrow R(\mathbb{C})$ uma função intervalar. Diz-se que $F$ representa $f$ quando para todo $z=a+i b \in \mathbb{C}$ tal que $a \in A$ e $b \in B$, tem-se $f(z)=c+i d$ com $c \in C$ e $d \in D$, em que $F(Z)=C+i D$.

Observação 4. Para $z=a+i b \in \mathbb{C}$, denota-se a parte real e imaginária do número complexo z por $\operatorname{Re}(z)=a$ e $\operatorname{Im}(z)=b$, respectivamente. Analogamente, para $Z=A+i B \in R(\mathbb{C})$, denota-se a parte real e imaginária do número complexo intervalar $Z$ por $\operatorname{Re}(Z)=A$ e $\operatorname{Im}(Z)=B$, respectivamente.

Lema 4.1. Seja $f: \mathbb{C} \rightarrow \mathbb{C}$ uma função complexa limitada em todo retângulo do plano complexo, isto é, se $R$ é um retângulo no plano complexo, então existe $0<K<\infty$ tal que $|f(z)|=\sqrt{\operatorname{Re}(f(z))^{2}+\operatorname{Im}(f(z))^{2}}<K$, para todo $z \in R$. A função intervalar $\widehat{f}: R(\mathbb{C}) \rightarrow R(\mathbb{C})$ definida por $\widehat{f}(A+i B)=[\underline{a}, \bar{a}]+i[\underline{b}, \bar{b}]$, em que $\underline{a}=\min \{\operatorname{Re}(f(a+i b)) \mid a \in A$ e $b \in B\}, \bar{a}=\max \{\operatorname{Re}(f(a+i b)) \mid a \in A$ e $b \in B\}$, $\underline{b}=\min \{\operatorname{Im}(f(a+i b)) \mid a \in A$ e $b \in B\}$ e $\bar{b}=\max \{\operatorname{Im}(f(a+i b)) \mid a \in A$ e b $\in$ B $\}$, é uma representação intervalar de $f$. 


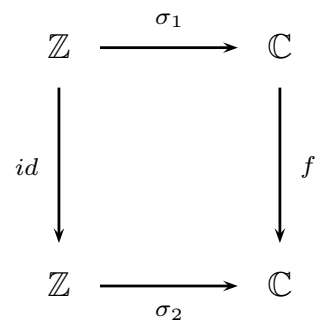

Figura 2: Sistema complexo.

Demonstração. Como $f$ é limitada em todo retângulo do plano complexo, temos que a função $\widehat{f}$ está bem definida. O fato de que $\widehat{f}$ representa $f$ é imediato.

Definição 4.10 (Representação Canônica Intervalar Complexa). Chama-se a função $\widehat{f}$, dada na Proposição 4.1, de representação canônica intervalar complexa $(C I R)$.

Teorema 4.1. Se $f: \mathbb{C} \rightarrow \mathbb{C}$ é uma função complexa limitada em todo retângulo do plano complexo, então para cada $A+i B \in R(\mathbb{C}), \widehat{f}(A+i B)$ é o menor retângulo que contém $f(z)$, para todo $z=a+i b$, com $a \in A$ e $b \in B$.

Demonstração. Seja $A+i B \in R(\mathbb{C})$. Tome um retângulo $R=\{z \in \mathbb{C} \mid z=c+i d, \underline{c} \leq$ $c \leq \bar{c}, \underline{d} \leq d \leq \bar{d}\}$, tal que $f(z) \in R$ para todo $z=a+i b$, com $a \in A$ e $b \in B$. Para todo $z=x+i y \in \mathbb{C}$, pode-se definir $f(z)=u(z)+i v(z)$, em que $u$ e $v$ são funções de $\mathbb{C}$ em $\mathbb{R}$. Como $f$ é uma função limitada em todo retângulo do plano complexo, as funções $u$ e $v$ também as são, portanto estas assumem máximo e mínimo em todo retângulo, em particular em $A+i B \in R(\mathbb{C})$. Agora, defina $\underline{a}=\min \{\operatorname{Re}(f(a+i b)) \mid a \in A, b \in B\}, \bar{a}=\max \{\operatorname{Re}(f(a+i b)) \mid a \in A, b \in B\}$, $\underline{b}=\min \{\operatorname{Im}(f(a+i b)) \mid a \in A, b \in B\}$ e $\bar{b}=\max \{\operatorname{Im}(f(a+i b)) \mid a \in A, b \in B\}$. Assim, para todo $x, y$ tal que $\underline{a} \leq x \leq \bar{a}$ e $\underline{b} \leq y \leq \bar{b}$, tem-se que $x+i y \in R$ e, consequentemente, $[\underline{a}, \bar{a}]+i[\underline{b}, \bar{b}] \subset R$. Logo, pela Definição 4.10, segue que $\widehat{f}(A+i B) \subset R$.

Seja $[\mathbb{Z} \rightarrow \mathbb{C}]$ a classe dos sinais de valores complexos em tempo discreto. Um sistema pode ser visto como uma operação que transforma sinais em sinais, i.e. uma função da forma $f:[\mathbb{Z} \rightarrow \mathbb{C}] \rightarrow[\mathbb{Z} \rightarrow \mathbb{C}]$. Entretanto o mesmo pode ser visto, também, como uma função $f: \mathbb{C} \rightarrow \mathbb{C}$ que faz o diagrama da Figura 2 comutar, i.e., $\forall n \in \mathbb{Z}, f\left(\sigma_{1}(n)\right)=\sigma_{2}(n)$.

Esta última abordagem é que será utilizada neste trabalho, no sentido que as funções $f: \mathbb{C} \rightarrow \mathbb{C}$ do diagrama serão estendidas para as suas respectivas representações canônicas intervalares $\widehat{f}: I(\mathbb{C}) \rightarrow I(\mathbb{C})$.

No que se segue, o sistema $f: \mathbb{C} \rightarrow \mathbb{C}, y[n]=f(x[n])$, será estendido a um sistema intervalar complexo, com o uso da representação canônica $\widehat{f}$. Será mostrado que as propriedades de causalidade, estabilidade, invariância no tempo, aditividade, homogeneidade e linearidade são preservadas. 
Proposição 4.1. Se $y[n]=f(x[n])$ é um sistema causal, então $Y[n]=\widehat{f}(X[n])$ é um sistema intervalar causal.

Demonstração. Seja $f$ um sistema causal, isto é, se $x_{1}[n]=x_{2}[n]$ para $n \leq n_{0}$, então $f\left(x_{1}[n]\right)=f\left(x_{2}[n]\right)$, para $n \leq n_{0}$. Tome dois sinais $X_{1}[n]=A[n]+i B[n], X_{2}[n]=$ $C[n]+i D[n] \in R(\mathbb{C})$, tais que $X_{1}[n]=X_{2}[n]$, para $n \leq n_{0}$. Isto é, $A[n]=C[n]$ e $B[n]=D[n]$, para $n \leq n_{0}$. Pelo Lema 4.1, segue que $\widehat{f}\left(X_{1}[n]\right)=\left[\underline{a_{n}}, \overline{a_{n}}\right]+i\left[\underline{b_{n}}, \overline{b_{n}}\right]$, em que:

$\underline{a_{n}}=\min \left\{\operatorname{Re}\left(f\left(a_{n}+i b_{n}\right)\right) \mid a_{n} \in A[n], b_{n} \in B[n]\right\} ;$

$\overline{\overline{a_{n}}}=\max \left\{\operatorname{Re}\left(f\left(a_{n}+i b_{n}\right)\right) \mid a_{n} \in A[n], b_{n} \in B[n]\right\} ;$

$\underline{b_{n}}=\min \left\{\operatorname{Im}\left(f\left(a_{n}+i b_{n}\right)\right) \mid a_{n} \in A[n], b_{n} \in B[n]\right\} ;$

$\overline{\overline{b_{n}}}=\max \left\{\operatorname{Im}\left(f\left(a_{n}+i b_{n}\right)\right) \mid a_{n} \in A[n], b_{n} \in B[n]\right\}$. Por outro lado, $\widehat{f}\left(X_{2}[n]\right)=$ $\left[\underline{c_{n}}, \overline{c_{n}}\right]+i\left[\underline{d_{n}}, \overline{d_{n}}\right]$, em que:

$\underline{\underline{c_{n}}}=\min \left\{\overline{\operatorname{Re}}\left(f\left(c_{n}+i d_{n}\right)\right) \mid c_{n} \in C[n], d_{n} \in D[n]\right\}$

$\overline{\overline{c_{n}}}=\max \left\{\operatorname{Re}\left(f\left(c_{n}+i d_{n}\right)\right) \mid c_{n} \in C[n], d_{n} \in D[n]\right\} ;$

$\underline{d_{n}}=\min \left\{\operatorname{Im}\left(f\left(c_{n}+i d_{n}\right)\right) \mid c_{n} \in C[n], d_{n} \in D[n]\right\} ;$

$\overline{\overline{d_{n}}}=\max \left\{\operatorname{Im}\left(f\left(c_{n}+i d_{n}\right)\right) \mid c_{n} \in C[n], d_{n} \in D[n]\right\}$.

Como $A[n]=C[n]$ e $B[n]=D[n]$, para $n \leq n_{0}$, tem-se $\underline{a_{n}}=\underline{c_{n}}, \overline{a_{n}}=\overline{c_{n}}, \underline{b_{n}}=\underline{d_{n}} \mathrm{e}$ $\overline{b_{n}}=\overline{d_{n}}$, para $n \leq n_{0}$. Logo, $\widehat{f}\left(X_{1}[n]\right)=\widehat{f}\left(X_{2}[n]\right)$, para $n \leq \overline{n_{0}}$.

Proposição 4.2. Se $y[n]=f(x[n])$ é um sistema estável, então $Y[n]=\widehat{f}(X[n])$ é um sistema complexo intervalar estável.

Demonstração. Tome $X[n]=A[n]+i B[n]$ limitado, em que $A[n]=\left[\underline{a}_{n}, \bar{a}_{n}\right]$ e $B[n]=\left[\underline{b}_{n}, \bar{b}_{n}\right]$. Então existem $0<K_{1}, K_{2}<\infty$ tais que $\max \left\{\left|\underline{a}_{n}\right|,\left|\bar{a}_{n}\right|\right\} \leq K_{1}$ e $\max \left\{\left|\underline{b}_{n}\right|,\left|\bar{b}_{n}\right|\right\} \leq K_{2}$. Assim, para todo $a_{n} \in A[n]$ e $b_{n} \in B[n]$ temos que $\left|a_{n}+i b_{n}\right|=$ $\max \left\{\left|a_{n}\right|,\left|b_{n}\right|\right\} \leq \max \left\{K_{1}, K_{2}\right\}$. Como $f$ é estável, isso implica em $\left|f\left(a_{n}+i b_{n}\right)\right|=$ $\left|c_{n}+i d_{n}\right|=\max \left\{\left|c_{n}\right|,\left|d_{n}\right|\right\} \leq K_{3}$, para um dado $0<K_{3}<\infty$. Consequentemente, fazendo $\underline{c}_{n}=\min \left\{\operatorname{Re}\left(f\left(a_{n}+i b_{n}\right)\right) \mid a_{n} \in A, b_{n} \in B\right\}, \bar{c}_{n}=\max \left\{\operatorname{Re}\left(f\left(a_{n}+i b_{n}\right)\right) \mid a_{n} \in\right.$ $\left.A_{n}, b_{n} \in B_{n}\right\}, \underline{d}_{n}=\min \left\{\operatorname{Im}\left(f\left(a_{n}+i b_{n}\right)\right) \mid a_{n} \in A_{n}, b_{n} \in B_{n}\right\}$ e $\bar{d}_{n}=\max \left\{\operatorname{Im}\left(f\left(a_{n}+\right.\right.\right.$ $\left.\left.\left.i b_{n}\right)\right) \mid a_{n} \in A_{n}, b_{n} \in B_{n}\right\}$, temos que $\max \left\{\left|\underline{c}_{n}\right|,\left|\bar{c}_{n}\right|\right\} \leq K_{3}$ e $\max \left\{\left|\underline{d}_{n}\right|,\left|\bar{d}_{n}\right|\right\} \leq K_{3}$. Logo, $\widehat{f}$ é estável.

Proposição 4.3. Se $y[n]=f(x[n])$ é um sistema invariante no tempo, então $Y[n]=\widehat{f}(X[n])$ é um sistema complexo intervalar invariante no tempo.

Demonstração. Para $X\left[n-n_{0}\right]=A\left[n-n_{0}\right]+i B\left[n-n_{0}\right]$, temos $\widehat{f}\left(X\left[n-n_{0}\right]\right)=$ $\left[\min \left\{\operatorname{Re}(f(a+i b)) \mid a \in A\left[n-n_{0}\right]\right.\right.$ e $\left.b \in B\left[n-n_{0}\right]\right\}, \max \{\operatorname{Re}(f(a+i b)) \mid a \in A[n-$ $\left.n_{0}\right]$ e $\left.\left.b \in B\left[n-n_{0}\right]\right\}\right]+i\left[\min \left\{\operatorname{Im}(f(a+i b)) \mid a \in A\left[n-n_{0}\right]\right.\right.$ e $\left.b \in B\left[n-n_{0}\right]\right\}, \max \{\operatorname{Im}(f(a+$ $i b)) \mid a \in A$ e $b \in B\}]$. Como $X\left[n-n_{0}\right]=A\left[n-n_{0}\right]+i B\left[n-n_{0}\right]$, para todo $a \in A\left[n-n_{0}\right]$ e $b \in B\left[n-n_{0}\right], f(a+i b)=y\left[n-n_{0}\right]$ pois $f$ é invariante no tempo. Logo, $\widehat{f}\left(X\left[n-n_{0}\right]\right)$ terá uma resposta com o mesmo deslocamento $n-n_{0}$, isto é, o sistema complexo intervalar é invariante no tempo. 
Proposição 4.4. Se $y[n]=f(x[n])$ é um sistema complexo aditivo, então $Y[n]=$ $\widehat{f}(X[n])$ é um sistema complexo intervalar aditivo.

Demonstração. Considere dois sinais complexos intervalares $X_{1}[n]=A_{1}+i B_{1}$ e $X_{2}[n]=A_{2}+i B_{2}$. Fazendo $\alpha_{1}=\left\{\operatorname{Re}\left(f\left(\left(a_{1}+i b_{1}\right)+\left(a_{2}+i b_{2}\right)\right)\right) \mid a_{1} \in A_{1}, b_{1} \in\right.$ $\left.B_{1}, a_{2} \in A_{2}, b_{2} \in B_{2}\right\}$ e $\alpha_{1}=\left\{\operatorname{Im}\left(f\left(\left(a_{1}+i b_{1}\right)+\left(a_{2}+i b_{2}\right)\right)\right) \mid a_{1} \in A_{1}, b_{1} \in\right.$ $\left.B_{1}, a_{2} \in A_{2}, b_{2} \in B_{2}\right\}$, temos que $\widehat{f}\left(X_{1}[n]+X_{2}[n]\right)=\left[\min \left\{\alpha_{1}\right\}, \max \left\{\alpha_{1}\right\}\right]+$ $i\left[\min \left\{\alpha_{2}\right\}, \max \left\{\alpha_{2}\right\}\right]$. Como $y[n]=f(x[n])$ é aditivo, $f\left(\left(a_{1}+i b_{1}\right)+\left(a_{2}+i b_{2}\right)\right)=$ $f\left(a_{1}+i b_{1}\right)+f\left(a_{2}+i b_{2}\right)$, então, fazendo $\beta_{1}=\left\{\operatorname{Re}\left(f\left(a_{1}+i b_{1}\right)\right) \mid a_{1} \in A_{1}, b_{1} \in\right.$ $\left.B_{1}\right\}, \beta_{2}=\left\{\operatorname{Re}\left(f\left(a_{2}+i b_{2}\right)\right) \mid a_{2} \in A_{2}, b_{2} \in B_{2}\right\}, \beta_{3}=\left\{\operatorname{Im}\left(f\left(a_{1}+i b_{1}\right)\right) \mid a_{1} \in\right.$ $\left.A_{1}, b_{1} \in B_{1}\right\}$ e $\beta_{4}=\left\{\operatorname{Im}\left(f\left(a_{2}+i b_{2}\right)\right) \mid a_{2} \in A_{2}, b_{2} \in B_{2}\right\}$, segue que $\widehat{f}\left(X_{1}[n]+\right.$ $\left.X_{2}[n]\right)=\left(\left[\min \left\{\beta_{1}\right\}, \max \left\{\beta_{1}\right\}\right]+i\left[\min \left\{\beta_{3}\right\}, \max \left\{\beta_{3}\right\}\right]\right)+\left(\left[\min \left\{\beta_{2}\right\}, \max \left\{\beta_{2}\right\}\right]+\right.$ $\left.i\left[\min \left\{\beta_{4}\right\}, \max \left\{\beta_{4}\right\}\right]\right)=\widehat{f}\left(X_{1}[n]\right)+\widehat{f}\left(X_{2}[n]\right)$, isto é, o sistema complexo intervalar $Y[n]=\widehat{f}(X[n])$ é aditivo.

Proposição 4.5. Se $y[n]=f(x[n])$ é um sistema complexo homogêneo, então $Y[n]=\widehat{f}(X[n])$ é um sistema complexo intervalar homogêneo.

Demonstração. Para $X[n]=A+i B \in R(\mathbb{C})$ e $k \in \mathbb{R}$, faça $\alpha_{1}=\{\operatorname{Re}(f(k(a+$ $i b))) \mid a \in A, b \in B\}$ e $\alpha_{2}=\{\operatorname{Im}(f(k(a+i b))) \mid a \in A, b \in B\}$. Assim, $\widehat{f}(k X[n])=$ $\left[\min \left\{\alpha_{1}\right\}, \max \left\{\alpha_{1}\right\}\right]+i\left[\min \left\{\alpha_{2}\right\}, \max \left\{\alpha_{2}\right\}\right]$. Como $f$ é homogêneo, $f(k(a+i b))=$ $k f(a+i b)$. Fazendo $\beta_{1}=\{\operatorname{Re}(f(a+i b))\}$ e $\beta_{2}=\{\operatorname{Im}(f(a+i b))\}$, temos $\widehat{f}(k X[n])=$ $\left[\min \left\{k \beta_{1}\right\}, \max \left\{k \beta_{1}\right\}\right]+i\left[\min \left\{k \beta_{2}\right\}, \max \left\{k \beta_{2}\right\}\right]=k\left(\left[\min \left\{\beta_{1}\right\}, \max \left\{\beta_{1}\right\}\right]+i\left[\min \left\{\beta_{2}\right\}\right.\right.$, $\left.\left.\max \left\{\beta_{2}\right\}\right]\right)=k \widehat{f}(X[n])$, isto é, o sistema complexo intervalar é homogêneo.

Proposição 4.6. Se $y[n]=f(x[n])$ é um sistema complexo linear, então $Y[n]=$ $\widehat{f}(X[n])$ um sistema complexo intervalar linear.

Demonstração. A demonstração segue imediatamente das Proposições 4.4 e 4.5.

\section{Conclusões}

Neste trabalho a aritmética complexa retangular foi aplicada ao contexto de processamento de sinais, com o objetivo de se obter uma fundamentação matemática para sinais e sistemas intervalares complexos. Foram definidas as principais classes de sistemas intervalares complexos, como a dos sistemas invariantes no tempo, estáveis e lineares. Também o conceito de representação canônica intervalar foi estendido para o caso complexo. Esta função foi utilizada para estender sistemas usuais a sistemas intervalares complexos e verificou-se que esta extensão preserva as propriedades mais relevantes do sistema clássico.

Os conceitos de sinais e sistemas intervalares para o caso de natureza complexa, introduzidos nesse trabalho, assim como suas propriedades, podem ser aplicados em problemas práticos, pois existem muitos sinais, como os de radiofrequência, que 
assumem valores complexos e necessitam de um tratamento da imprecisão existente no mesmo. Assim, a fundamentação matemática desses conceitos possibilita a investigação de várias ferramentas e algoritmos para o processamento digital de sinais.

Em trabalhos futuros, pretendemos definir as transformadas $\mathcal{Z}$ e de Fourier, que são muito utilizadas no processamento digital de sinais, no caso complexo e estudar suas propriedades.

\section{Referências}

[1] G. Alefeld, J. Herzberger, "Introduction to Interval Computations", Academic Press, New York, 1983.

[2] M. Buslowicz, T. Kaczorek, Robust stability of positive discrete-time interval systems with time-delays. Bulletin of the Polish Academy of Sciences, Technical Sciences, 52, No. 2 (2004).

[3] M.M.C. Cruz, R.H.N. Santiago, A.D. Dória Neto, Mathematical morphology for two valued gray-scale images with undefined information, In: "Proceedings of the 8th International Symposium on Mathematical Morphology", 2008, Rio de Janeiro, RJ, 1 (2008), 1-6.

[4] W. Edmonson, W.H. Lee, J.M.M. Anderson, Interval methods for sinusoidal parameter estimation: A comparative analysis, Reliable Computing, 6 (2000), 321-336.

[5] W. Edmonson, R. Gupte, S. Ocloo, J. Gianchandani, W. Alexander, Interval Arithmetic Logic Unit for Signal Processing and Control Applications. In: Proceedings of the NSF Workshop on Reliable Engineering Computing: Modeling Errors and Uncertainty in Engineering Computations, (R.L. Muhanna, R.L. Mullen, eds.), Savannah, Georgia, 189-196, 2006.

[6] S. Haykin, B.V. Veen, "Sinais e Sistemas", Porto Alegre, RS, Bookman, 2001.

[7] T.J. Hickey, Qun Ju, M.H. van Emden, Interval arithmetic: from principles to implementation, Journal of the ACM, 48 (2001), 1038-1068.

[8] U. Kulisch, W. Miranker, "Computer Arithmetic in Theory and Practice", New York, Academic Press, 1981.

[9] B.P. Lathi. "Sinais e Sistemas Lineares", 2.ed., Porto Alegre, RS, Bookman, 2007.

[10] A. Lyra, B.R.C. Bedregal, R.C. Bedregal, A.D. Dória Neto, The interval digital images processing, WSEAS Transactions on Circuits, 3 234-240, 2004.

[11] S. Markov, On direct interval arithmetic and its applications. J. Universal Computer Science, 1, No.7 (1995), 514-526.

[12] R.E. Moore, "Interval Analysis", Prentice Hall, New Jersey, 1966. 
[13] R.E. Moore, "Methods and Applications of Interval Analysis", Studies in Applied Mathematics-SIAM, Philadelphia, 1979.

[14] M. Mukaidomo, "Fuzzy Logic for Beginners", World Scientific, 2004.

[15] A.V. Oppenheim, R.W. Schafer, "Discrete Time Signal Processing", Prentice Hall, 1989.

[16] M. Petković, L. Petković, "Complex Intervalar Arithmetic and its Applications", Mathematical Research, Vol. 105, 1.ed., Berlin, Wiley-Vch, 1998.

[17] J. Rokne; P. Lancaster, "Complex Interval Arithmetic", Numerical Mathematics. (W.P. Timlac, ed.), 1970.

[18] F.T. Santana, R.H.N. Santiago, A.D. Dória Neto, Fundamentação intervalar complexa para sinais e sistemas. In: XXXIII Congresso Nacional de Matemática Aplicada e Computacional, 2010, Águas de Lindóia. Anais do XXXIII Congresso Nacional da Sociedade Brasileira de Matemática Aplicada e Computacional. São Carlos: SBMAC, 3 (2009), 1-7.

[19] F.T. Santana, F.L. Santana, A.M.G. Guerreiro, A.D. Dória Neto, R.H.N. Santiago. A framework for interval quantization and application to interval based algorithms in digital signal processing, Fundamenta Informaticae, 112 (2011), $337-363$.

[20] R.H.N. Santiago, B.R.C. Bedregal, B.M. Acióly, Formal aspects of correctness and optimality of interval computations, Formal Aspects of Computing, 18 (2006), 231-243.

[21] R.M.P. Trindade, B.R.C. Bedregal, A.D. Dória Neto, Princípios de processamento digital de sinais intervalares, TEMA - Tendências em Matemática Aplicada e Computacional, 10 (2009), 87-97.

[22] R.M.P. Trindade, R.R. . Bedregal, A.D. Dória Neto, Basic concepts of intervalar digital signal processing, International Journal of Electrical, Computer, and Systems Engineering, 4 (2010), 135-139. 